\title{
Eicosapentaenoic acid for prevention of major coronary events
}

\author{
Claudio Galli $^{\text {a, }}$, Patrizia Risé ${ }^{a}$, Cesare Sirtori ${ }^{\text {a }}$ \\ ${ }^{a}$ Department of Pharmacological Sciences, University of Milan, Via Balzaretti 9, 20133 Milan, Italy
}

\begin{abstract}
Mitsohiro Yokohama and colleagues ${ }^{1}$ noted that non-fatal coronary events, but not cardiac deaths, were lower in hypercholesterolaemic Japanese patients who were given $1800 \mathrm{mg}$ per day of the omega 3 fatty acid eicosapentaenoic acid (EPA) than in controls. At first, these results seem to contrast with other published data which indicate that omega 3 fatty acid preparations, including the end product of this metabolic series, docosahexaenoic acid (DHA), also reduce cardiac death. ${ }^{2}$ However, these findings are expected, given the chemistry and metabolism of EPA versus DHA.
\end{abstract}

EPA, by contrast with DHA, is not incorporated very efficiently into cell phospholipids (including cardiac myocytes). Since the antiarrhythmic effects, and the reduction of cardiac mortality, of omega 3 are related to the incorporation of these fatty acids into heart phospholipids, the effects of EPA would be lower than those of DHA. ${ }^{3,4}$

No data on basal concentrations of omega 3 fatty acids were presented. They should be high compared with those in Western populations. After treatment, EPA concentrations in plasma lipids should increase, while those of DHA should not. In fact, they should be high already owing to fish intake, and the conversion of EPA to DHA, rather inefficient under conventional dietary conditions, should be even lower in the presence of high levels of DHA. ${ }^{5}$ Data on fatty acid profiles before and after treatment would facilitate interpretations.

EPA inhibits the production of proinflammatory eicosanoids more efficiently than DHA, since EPA competes with the omega 6 fatty acid arachidonic acid for oxygenases. Non-fatal coronary events, possibly related to inflammatory processes, should thus be best counteracted by EPA.

We declare that we have no conflict of interest.

\section{References}

1. Yokoyama M, Origasa $\mathrm{H}$, Matsuzaki $\mathrm{M}$, et al. for the Japan EPA lipid intervention study (JELIS) InvestigatorsEffects of eicosapentaenoic acid on major coronary events in hypercholesterolaemic patients (JELIS): a randomised open-label, blinded endpoint analysis, Lancet, Volume: 369, (2007), pp. 1090-1098

2. Kris-Etherton PM, Harris WS, Appel LJ, et al. Fish consumption, fish oil, omega-3 fatty acids, and cardiovascular disease, Circulation, Volume: 106, (2002), pp. 2747-2757

3. Harris WS, Sands SA, Windsor SL, et al. Omega-3 fatty acids in cardiac biopsies from heart transplantation patients, Circulation, Volume: 110, (2004), pp. 1645-1649

4. Mori TA, Woodman RJ, The independent effects of eicosapentaenoic acid and docosahexaenoic acid on cardiovascular risk factors in humans, Curr Opin Clin Nutr Metab Care, Volume: 9, (2006), pp. 95104

5. Burdge GC, Calder PC, Conversion of a-linolenic acid to longer-chain polyunsaturated fatty acids in human adults, Reprod Nutr Dev, Volume: 45, (2005), pp. 581--597 